Docent dr Miljko Popović, pukovnik, dipl. inž. Vojna akademija, Beograd

\section{BORBENI ZAOKRET AVIONA}

UDC: $623.746 .34: 627.7 .07$

Rezime:

U radu su prikazane jednačine kretanja težišta aviona u borbenom zaokretu $i$ analiza uticaja koeficijenta opterećenja i ugla naginjanja na karakteristike borbenog zaokreta, kao što su: brzina, promena ugla nagiba putanje, prirast visine i vreme trajanja zaokreta.

Ključne reči: borbeni zaokret, koeficijent opterećenja, ugao naginjanja, ugao skretanja, aerodinamička sila, potisak.

\title{
COMBAT TURN
}

Summary:

The paper presents equations of motion of the aircraft center of mass in combat turn and effects of load factor and the bank angle on the characteristic of combat turn, such as: velocity, the flight path angle, increment of altitude and the time combat turn.

Key words: combat turn, load factor, bank angle, heading angle, aerodynamic force, thrust.

\section{Uvod}

Kretanje aviona $u$ prostornom manevru može se analizirati na više načina, zavisno od toga u kojem koordinatnom sistemu se rešavaju jednačine kretanja. Izborom polubrzinskog koordinatnog sistema za rešavanje jednačina kretanja obezbeđuje se najjednostavnije dolaženje do karakteristika borbenog zaokreta, kao što su: promena brzine, prirast visine, promena ugla nagiba putanje $u$ odnosu na horizont i vreme trajanja zaokreta. Do sada, borbeni zaokret aviona razmatran je, nepotpuno, $\mathrm{u}[1]$, a horizontalni zaokret $\mathrm{u}$ [3].

\section{Definicija borbenog zaokreta $i$ jednačine kretanja}

Borbeni zaokret bez klizanja jeste neustaljeni prostorni manevar aviona, pri kojem se menja pravac leta i istovremeno povećava visina. Borbeni zaokret obično se razmatra kao zaokret za $180^{\circ}$. Ovaj manevar najčešće se koristi u vazdušnoj borbi, kada se nastoji da se protivniku dođe iza leđa, i to, po mogućnosti, sa nadvišenjem. Prednost u visini je gotovo uvek poželjna, jer se potencijalna energija može brzo pretvoriti u kinetičku i tako postići željena brzina.

\section{Izbor koordinatnog sistema za rešavanje jednačina kretanja}

Da bismo potpuno definisali koordinatni sistem, potrebno je odrediti pravac ose Oz. U dinamici leta uobičajeno je da se ona nalazi bilo u ravni simetrije, bilo $u$ vertikalnoj ravni.

U prvom slučaju koordinatni sistem nazivamo brzinskim, $X_{v}, Y_{v}, Z_{v}$, a u dru- 
gom polubrzinskim, $\mathrm{X}_{\mathrm{v}}, \mathrm{Y}_{*}, \mathrm{Z}_{*}$, kako je prikazano na sl. 1. Dakle, kod polubrzinskog koordinatnog sistema osa $\mathrm{OZ}_{*}$ tokom kretanja aviona uvek ostaje $\mathrm{u}$ vertikalnoj ravni, a osa $\mathrm{OY} *$ je uvek horizontalna, što pojednostavljuje rešavanje jednačina kretanja. Prema tome, položaj polubrzinskog koordinatnog sistema $\mathrm{u}$ odnosu na sistem lokalnog horizonta određuje se jedino pravcem ose $\mathrm{OX}_{\mathrm{v}}$ tj. uglovima $\chi$ i $\gamma$.

Kretanje centra masa aviona može se izraziti u polubrzinskom koordinatnom sistemu. Opšta jednačina kretanja centra masa aviona u proizvoljnom koordinatnom sistemu glasi:

$m \frac{d \vec{V}}{d t}=\vec{R}+\vec{T}+\vec{G}$ gde su:

$\mathrm{m}$ - masa aviona,

$\overrightarrow{\mathrm{V}}$ - vektor brzine,

$\overrightarrow{\mathrm{R}}$ - aerodinamička sila,

$\overrightarrow{\mathrm{T}}$ - sila potiska,

$\overrightarrow{\mathrm{G}}=\mathrm{m} \cdot \overrightarrow{\mathrm{g}}-$ težina aviona $\mathrm{i}$

$\frac{\mathrm{d} \overrightarrow{\mathrm{V}}}{\mathrm{dt}}$ - izvod brzine po vremenu (apsolutno ubrzanje tačke).

Izvod vektora brzine u rotirajućem koordinatnom sistemu osa sa ugaonom brzinom $\vec{\omega}$ je: $\frac{d \vec{V}}{d t}=\frac{\partial \vec{V}}{\partial t}+\vec{\omega} \times \vec{V}$, pa gornja jednačina primenjena na polubrzinski koordinatni sistem postaje:

$m \frac{\partial \vec{V}}{d t}+m \cdot \vec{\omega}^{*} \times \vec{V}=\vec{R}+\vec{T}+m \cdot \vec{g}$

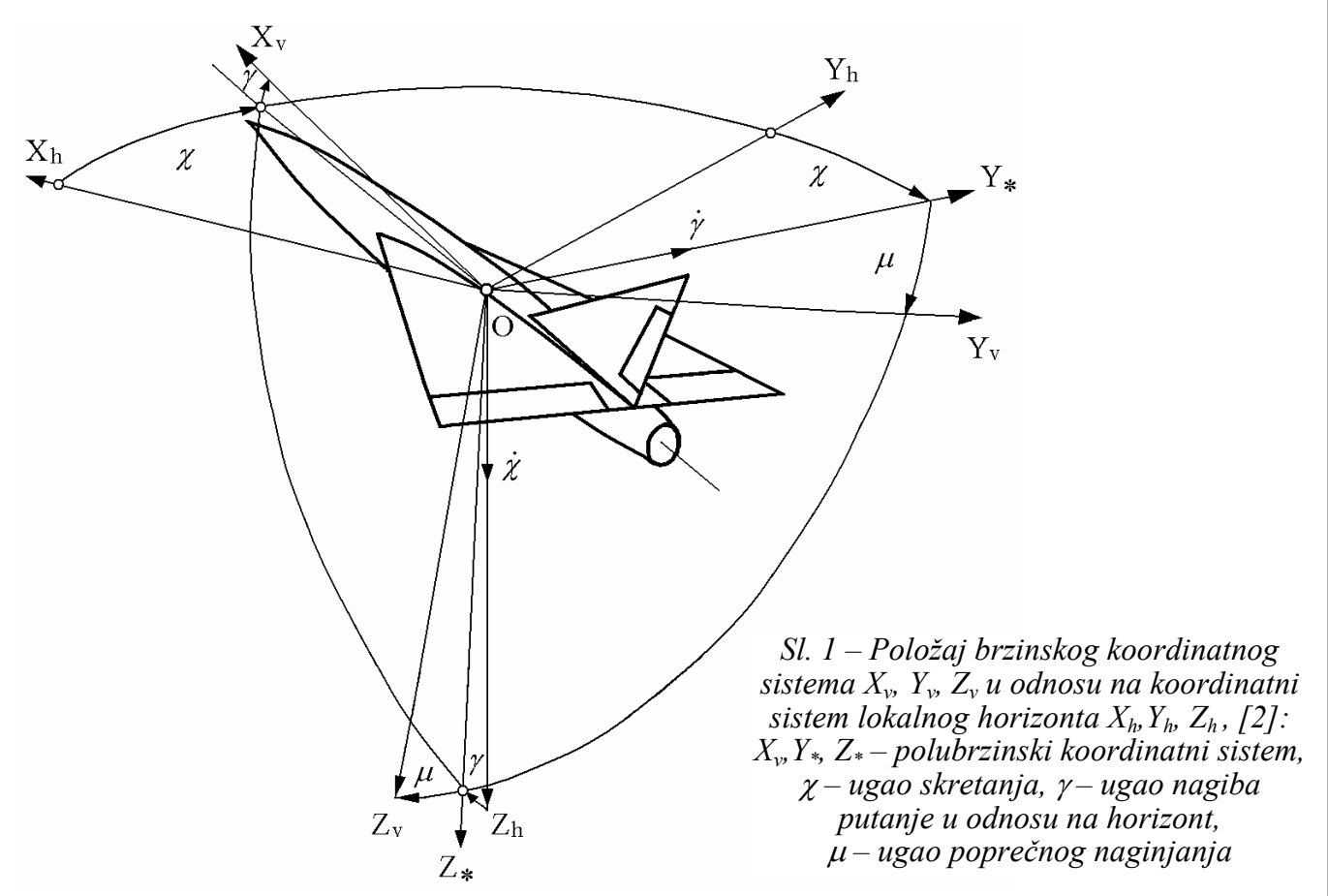


gde je:

$\vec{\omega}^{*}$ - ugaona brzina obrtanja polubrzinskog koordinatnog sistema u odnosu na koordinatni sistem prividnog horizonta.

Radi razvoja jednačina kretanja težišta aviona $\mathrm{u}$ polubrzinskom koordinatnom sistemu potrebno je izvršiti neophodne transformacije.

Transformacija iz koordinatnog sistema lokalnog horizonta u polubrzinski koordinatni sistem ostvaruje se kroz dve sukcesivne jednoosne rotacije, $i$ to:

- rotacijom oko ose $\mathrm{OZ}_{\mathrm{h}}$ koordinatnog sistema lokalnog horizonta za ugao $\chi$ (sl. 1) koja je definisana matricom transformacije $\mathrm{C}_{1}$;

- rotacijom oko novostvorene ose $\mathrm{OY}_{*}$ za ugao $\gamma$ (sl. 1), koja je definisana matricom transformacije $\mathrm{C}_{2}$,

gde su matrice transformacija jednoosnih rotacija:

$$
\begin{aligned}
& C_{1}=\left[\begin{array}{ccc}
\cos \chi & \sin \chi & 0 \\
-\sin \chi & \cos \chi & 0 \\
0 & 0 & 1
\end{array}\right] \\
& C_{2}=\left[\begin{array}{ccc}
\cos \gamma & 0 & -\sin \gamma \\
0 & 1 & 0 \\
\sin \gamma & 0 & \cos \gamma
\end{array}\right]
\end{aligned}
$$

Matrica transformacije iz koordinatnog sistema lokalnog horizonta u polubrzinski koordinatni sistem jednaka je proizvodu sukcesivnih jednoosnih matrica transformacija:

$$
\begin{aligned}
& C_{h}^{p b}=C_{2} \cdot C_{1}= \\
& =\left[\begin{array}{ccc}
\cos \gamma \cos \chi & \cos \gamma \sin \chi & -\sin \gamma \\
-\sin \chi & \cos \chi & 0 \\
\sin \gamma \cos \chi & \sin \gamma \sin \chi & \cos \gamma
\end{array}\right]
\end{aligned}
$$

Transformacija iz brzinskog u polubrzinski koordinatni sistem ostvaruje se jednoosnom rotacijom oko ose $\mathrm{OX}_{\mathrm{v}}$ brzinskog koordinatnog sistema za ugao $\mu$, (sl. 1). U daljem tekstu osa $\mathrm{OX}_{\mathrm{v}}$ obeležena je sa OX.

Matrica transformacije brzinskog $\mathrm{u}$ polubrzinski koordinatni sistem je:

$$
C_{v}^{p b}=\left[\begin{array}{ccc}
1 & 0 & 0 \\
0 & \cos \mu & -\sin \mu \\
0 & \sin \mu & \cos \mu
\end{array}\right]
$$

Matrični oblik izraza (2) za polubrzinski koordinatni sistem $\mathrm{X}_{\mathrm{v}}=\mathrm{X}, \mathrm{Y}_{*}, \mathrm{Z} *$ glasi:

$m\left[\begin{array}{c}\dot{V}_{x} \\ \dot{V}_{y^{*}} \\ \dot{V}_{z^{*}}\end{array}\right]+m\left[\begin{array}{ccc}0 & -\omega_{z}^{*} & \omega_{y}^{*} \\ \omega_{z}^{*} & 0 & -\omega_{x} \\ -\omega_{y}^{*} & \omega_{x} & 0\end{array}\right]$.

$\cdot\left[\begin{array}{c}V_{x} \\ V_{y^{*}} \\ V_{z^{*}}\end{array}\right]=\left[\begin{array}{c}F_{x} \\ F_{y^{*}} \\ F_{z^{*}}\end{array}\right]$

gde su:

$\omega_{\mathrm{x}}, \omega_{\mathrm{y}}^{*} \mathrm{i} \omega_{\mathrm{z}}^{*}-$ komponente vektora ugaone brzine $u$ pravcu osa polubrzinskog koordinatnog sistema;

$V_{x}, V_{y^{*}}$ i $V_{z^{*}}$ - komponente vektora brzine u pravcu osa polubrzinskog sistema; $F_{x}, \mathrm{~F}_{\mathrm{y}^{*}}, \mathrm{~F}_{z^{*}}-$ projekcije svih spoljašnjih sila u pravcu osa polubrzinskog koordinatnog sistema.

U polubrzinskom koordinatnom sistemu su:

- komponente vektora ugaone brzine (odnosi su jasno uočljivi na sl. 1): 
$\omega_{x}=-\dot{\chi} \sin \gamma, \quad \omega_{y}^{*}=\dot{\gamma} \quad \omega_{z}^{*}=\dot{\chi} \cos \gamma$

- komponente vektora brzine:

$V_{x}=V, \quad V_{y^{*}}=0, \quad V_{z^{*}}=0$

\section{Komponente aerodinamičke sile}

Transformacija komponenti aerodinamičke sile iz brzinskog koordinatnog sistema $\mathrm{R}^{\mathrm{v}}=\left\{-\mathrm{R}_{\mathrm{x}}, \mathrm{R}_{\mathrm{y}},-\mathrm{R}_{\mathrm{z}}\right\}$ na ose polubrzinskog koordinatnog sistema $\mathrm{R}^{\mathrm{pb}}=\left\{-\mathrm{R}_{\mathrm{x}}, \mathrm{R}_{\mathrm{y}^{*}}, \mathrm{R}_{\mathrm{z}^{*}}\right\}$ vrši se pomoću matrice transformacije $\mathrm{C}_{\mathrm{v}}^{\mathrm{pb}}$, korišćenjem relacije:

$R^{p b}=C_{v}^{p b} \cdot R^{v}$

odnosno:

$$
\begin{aligned}
& {\left[\begin{array}{c}
R_{x} \\
R_{y^{*}} \\
R_{z^{*}}
\end{array}\right]=\left[\begin{array}{ccc}
1 & 0 & 0 \\
0 & \cos \mu & -\sin \mu \\
0 & \sin \mu & \cos \mu
\end{array}\right] \cdot\left[\begin{array}{c}
-R_{x} \\
0 \\
-R_{z}
\end{array}\right]=} \\
& =\left[\begin{array}{c}
-R_{x} \\
R_{z} \sin \mu \\
-R_{z} \cos \mu
\end{array}\right]
\end{aligned}
$$

Komponente sile masa u pravcu osa polubrzinskog sistema

Komponente sile masa u pravcu osa polubrzinskog sistema iznose:

$$
G^{p b}=C_{2} \cdot G^{h}
$$

gde su:

$\mathrm{G}^{\mathrm{pb}}=\left\{\mathrm{G}_{\mathrm{x}}, \mathrm{G}_{\mathrm{y}^{*}}, \mathrm{G}_{\mathrm{z}^{*}}\right\}$ - komponente težine u polubrzinskom koordinatnom sistemu,
$\mathrm{G}^{\mathrm{h}}=\{0,0, \mathrm{mg}\}-$ komponente težine $\mathrm{u}$ koordinatnom sistemu lokalnog horizonta. Iz jednačine (5) dobija se:

$G_{x}=-m g \sin \gamma$,

$G_{y^{*}}=0$,

$G_{z^{*}}=m g \cos \gamma$.

\section{Komponente sile potiska}

Sila potiska najpre se projektuje na ose brzinskog koordinatnog sistema, gde su njene komponente $\mathrm{T}^{\mathrm{v}}=\left\{\mathrm{T}_{\mathrm{xv}}, \mathrm{T}_{\mathrm{yv}}, \mathrm{T}_{\mathrm{zv}}\right\}$ $=\left\{\mathrm{T} \cos \left(\alpha-\alpha_{\mathrm{s}}\right), 0,-\mathrm{T} \sin \left(\alpha-\alpha_{\mathrm{s}}\right)\right\}, \mathrm{i}$ nakon toga u polubrzinski sa komponentama $\mathrm{T}^{\mathrm{pb}}=\left\{\mathrm{T}_{\mathrm{x}}, \mathrm{T}_{\mathrm{y}^{*}}, \mathrm{~T}_{\mathrm{z}^{*}}\right\}$, pomoću relacije:

$T^{p b}=C_{v}^{p b} \cdot T^{v}$

Odavde se dobija:

$\left[\begin{array}{c}T_{x} \\ T_{y^{*}} \\ T_{z^{*}}\end{array}\right]=\left[\begin{array}{ccc}1 & 0 & 0 \\ 0 & \cos \mu & -\sin \mu \\ 0 & \sin \mu & \cos \mu\end{array}\right]$.

$\cdot\left[\begin{array}{c}T \cos \left(\alpha-\alpha_{s}\right) \\ 0 \\ -T \sin \left(\alpha-\alpha_{s}\right)\end{array}\right]=\left[\begin{array}{c}T \cos \left(\alpha-\alpha_{s}\right) \\ T \sin \left(\alpha-\alpha_{s}\right) \sin \mu \\ -T \sin \left(\alpha-\alpha_{s}\right) \cos \mu\end{array}\right]$

pri čemu je $\alpha_{\mathrm{s}}-$ smeštajni ugao krila aviona.

Tako su sada komponente svih spoljašnjih sila u pravcu osa polubrzinskog sistema:

$F_{x}=T \cos \left(\alpha-\alpha_{s}\right)-R_{x}-m g \sin \gamma$,

$F_{y}^{*}=\left[R_{z}+T \sin \left(\alpha-\alpha_{s}\right)\right] \cdot \sin \mu$,

$F_{z}^{*}=-\left[R_{z}+T \sin \left(\alpha-\alpha_{s}\right)\right] \cdot \cos \mu+m g \cos \gamma$. 
Nakon matričnog množenja izraza (3) i smene izraza za projekcije svih spoljašnjih sila u pravcu odgovarajućih osa, dolazi se do projekcija jednačina kretanja centra masa aviona u borbenom zaokretu na ose polubrzinskog sistema:

$m \frac{d V}{d t}=T \cos \left(\alpha-\alpha_{s}\right)-R_{x}-m g \sin \gamma$,

$\cos \gamma m V \frac{d \chi}{d t}=\left[R_{z}+T \sin \left(\alpha-\alpha_{s}\right)\right] \sin \mu$,

$m V \frac{d \gamma}{d t}=\left[R_{z}+T \sin \left(\alpha-\alpha_{s}\right)\right]$

(7)

$\cos \mu-m g \cos \gamma$

Dakle, kretanje aviona u borbenom zaokretu opisuju jednačine neustaljenog prostornog kretanja bez klizanja.

Nakon svih prethodnih razmatranja, sada se može grafički prikazati putanja aviona u toku borbenog zaokreta i sile koje deluju na težište aviona (sl. 2).

Uvođenjem koeficijenata tangencijalnog i normalnog opterećenja,

$$
\begin{aligned}
& n_{x}=\frac{T \cos \left(\alpha-\alpha_{s}\right)-R_{x}}{m g}, \\
& n=\frac{R_{z}+T \sin \left(\alpha-\alpha_{s}\right)}{m g}
\end{aligned}
$$

jednačine (7), sa dodatom kinematskom jednačinom $\mathrm{dh} / \mathrm{dt}=\mathrm{V} \sin \gamma$, postaju:

$$
\begin{aligned}
& \frac{d V}{d t}=g\left(n_{x}-\sin \gamma\right) \\
& \frac{d \chi}{d t}=\frac{g}{V \cos \gamma} n \sin \mu \\
& \frac{d \gamma}{d t}=\frac{g}{V}(n \cos \mu-\cos \gamma)
\end{aligned}
$$

$\frac{d h}{d t}=V \sin \gamma$

Sistem diferencijalnih jednačina (8) određuje uticaj koeficijenata normalnog i tangencijalnog opterećenja na promenu brzine $\mathrm{V}$, ugla nagiba putanje $\gamma$ i ugaone brzine $\mathrm{d} \chi / \mathrm{dt} \mathrm{u}$ toku izvođenja borbenog zaokreta, za datu vrednost parametra $\mu$.

Sl. 2 - Prikaz putanje aviona i sila koje deluju na avion u borbenom zaokretu

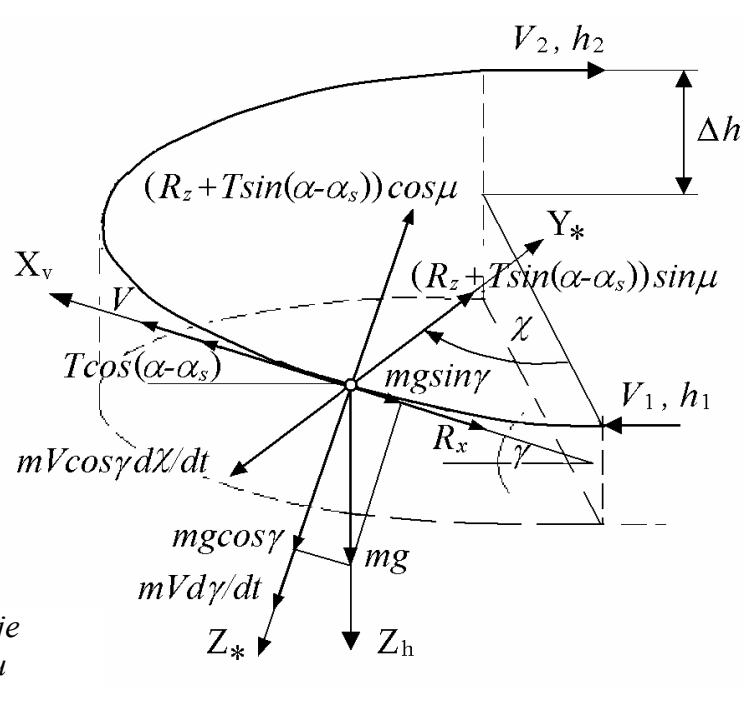


Koeficijenti opterećenja $n_{x}$ i $n$ menjaju se u toku izvođenja zaokreta. Međutim, može se pretpostaviti da su konstantni. Pri tome se pretpostavlja da od vrednosti $\mathrm{n}=1$ pilot na ulasku u zaokret trenutno prelazi na određeno $\mathrm{n} u$ samom zaokretu, i da se od njega opet trenutno vraća na $\mathrm{n}=1$ na završetku zaokreta.

Ako kao nezavisno promenljivu uvedemo ugao skretanja putanje $\chi$, nakon deljenja prve, treće i četvrte jednačine sistema (8) sa drugom jednačinom, dobijamo sistem jednačina:

$$
\begin{aligned}
& \frac{d V}{d \chi}=\frac{V \cos \gamma}{n \sin \mu}\left(n_{x}-\sin \gamma\right) \\
& \frac{d \gamma}{d \chi}=\frac{\cos \gamma}{n \sin \mu}(n \cos \mu-\cos \gamma) \\
& \frac{d h}{d \chi}=\frac{V^{2}}{g} \frac{\sin \gamma \cos \gamma}{n \sin \mu} \\
& \frac{d t}{d \chi}=\frac{V}{g} \frac{\cos \gamma}{n \sin \mu}
\end{aligned}
$$

Jednačine (9) određuju promenu brzine $\mathrm{V}$, ugla nagiba putanje $\gamma$ i visine $\mathrm{h} \mathrm{u}$ toku izvođenja borbenog zaokreta $\mathrm{u}$ zavisnosti od ugla skretanja putanje $\chi$, a sa $\mathrm{n}_{\mathrm{x}}, \mathrm{n}$ i $\mu$ kao parametrima. Dodata je i četvrta jednačina, koja neposredno sledi iz druge jednačine sistema (8), a koja određuje vreme $t$ borbenog zaokreta.

Moguća varijanta borbenog zaokreta je zaokret sa $n_{x}=0, t j$. kada je za sve vreme izvođenja zaokreta propulzivna sila $\mathrm{T}$ jednaka ili gotovo jednaka sili aerodinamičkog otpora $R_{\mathrm{x}}$.
Prirast visine $\Delta \mathrm{h}=\mathrm{h}_{2}-\mathrm{h}_{1}$, jedna od najvažnijih karakteristika borbenog zaokreta, dobija se iz energetske jednačine, tj. iz jednakosti promena potencijalne $\mathrm{i}$ kinetičke energije:

$$
\Delta h=\frac{V_{1}^{2}-V_{2}^{2}}{2 g}=\frac{V_{1}^{2}}{2 g}\left[1-\left(\frac{V_{2}}{V_{1}}\right)^{2}\right]
$$

\section{Rezultati proračuna}

Sistem diferencijalnih jednačina (9) rešen je u MATLAB-u korišćenjem metode Runge-Kutta četvrtog reda za brzinu ulaska u borbeni zaokret $V_{1}=310 \mathrm{~m} / \mathrm{s}$ sa korakom promene ugla skretanja $\Delta \chi=0,05 \mathrm{rad}$ i pri $\mathrm{n}_{\mathrm{x}}=0$.

Zavisnost $\mathrm{V}_{2} / \mathrm{V}_{1}=\mathrm{f}(\mathrm{n})$ prikazana je na sl. 3 za nekoliko vrednosti ugla naginjanja $\mu$.

Krive $\gamma_{2}=f(n)$, ugao nagiba putanje na izlasku iz borbenog zaokreta, prikazane su na sl. 4 za nekoliko vrednosti ugla poprečnog naginjanja $\mu$. Presečne tačke ovih krivih sa apscisom odgovaraju pravilnom horizontalnom zaokretu za koji je $n=1 / \cos \mu$.

Prirast visine $\Delta \mathrm{h}$ prikazan je na sl. $5 \mathrm{u}$ bezdimenzionalnom obliku $\eta=\Delta \mathrm{h}$ $\left(2 \mathrm{~g} / \mathrm{V}_{1}^{2}\right)$ [4], u zavisnosti od koeficijenta normalnog opterećenja za nekoliko vrednosti ugla poprečnog naginjanja $\mu$.

$\mathrm{Na}$ sl. 6 prikazan je uticaj koeficijenta normalnog opterećenja $n$ i ugla naginjanja $\mu$ na vreme zaokreta $u$ bezdimenzionalnom obliku $\tau=\mathrm{t} \cdot\left(\mathrm{g} / \mathrm{V}_{1}\right)$ [4]. 


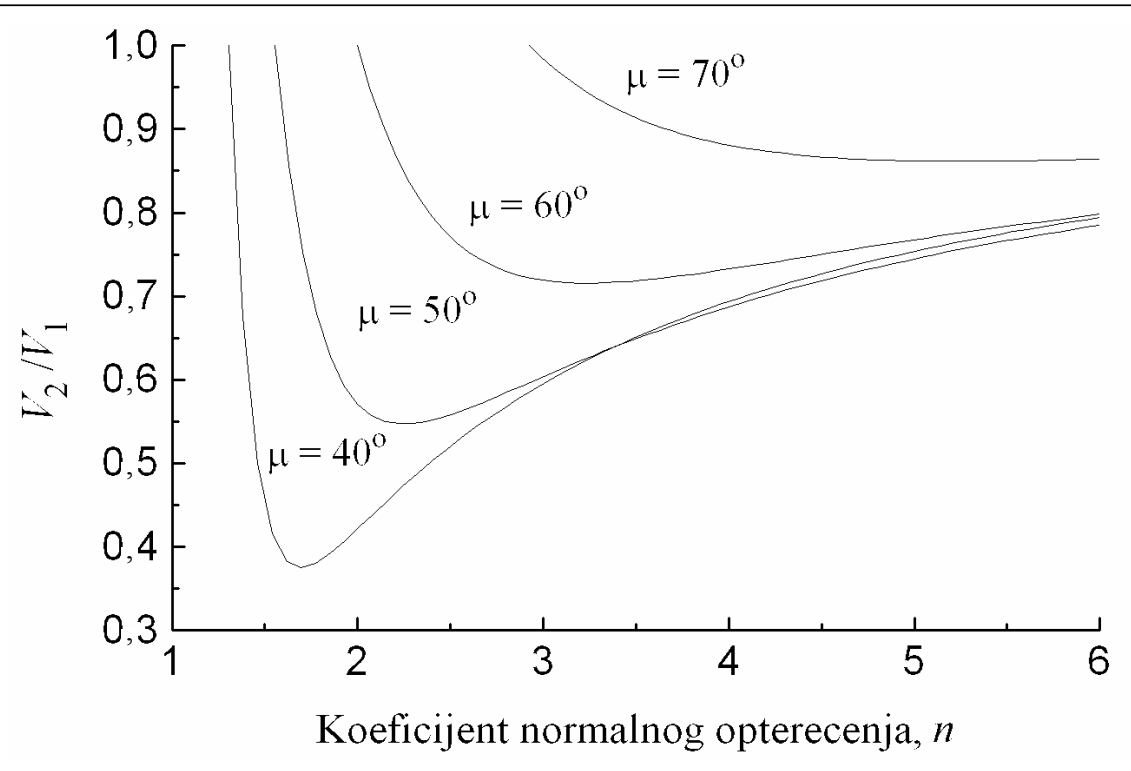

Sl. 3 - Odnos brzina na izlazu i ulazu borbenog zaokreta

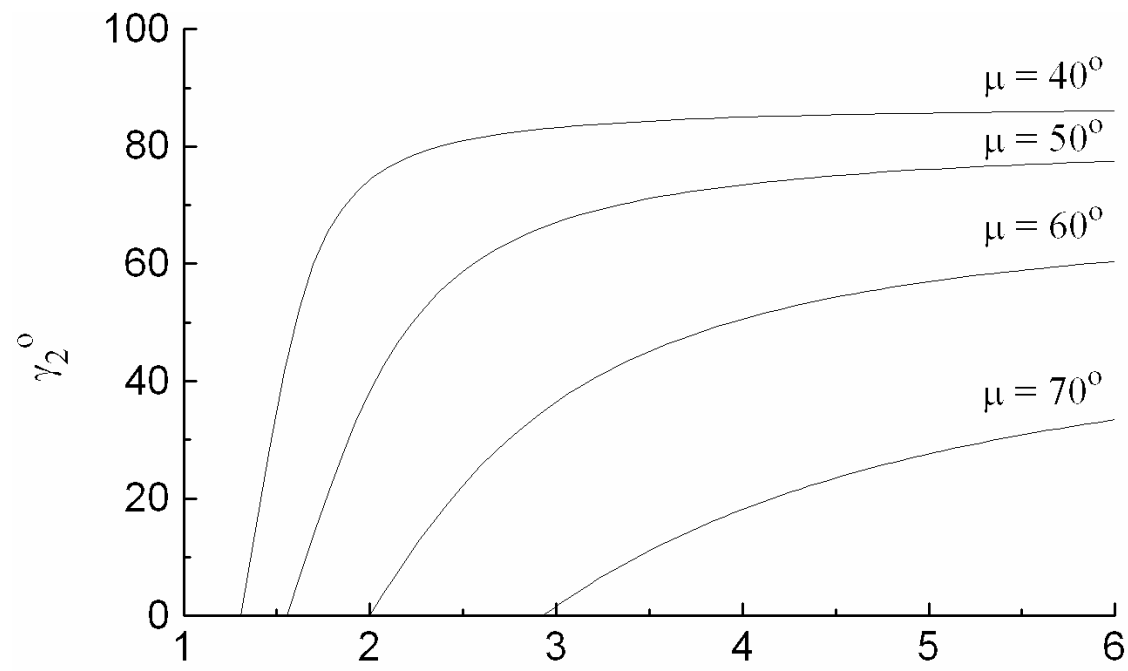

Koeficijent normalnog opterecenja, $n$

Sl. 4 - Ugao nagiba putanje na izlazu iz borbenog zaokreta 


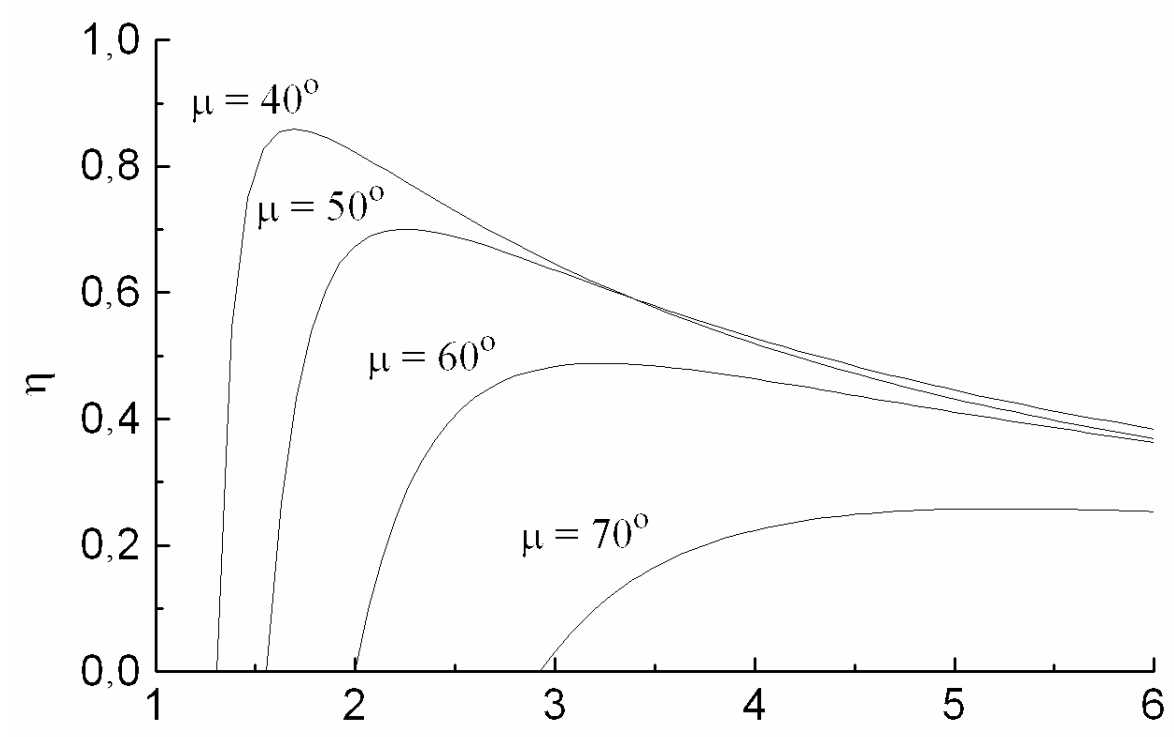

Koeficijent normalnog opterecenja, $n$

Sl. 5 - Prirast visine u borbenom zaokretu

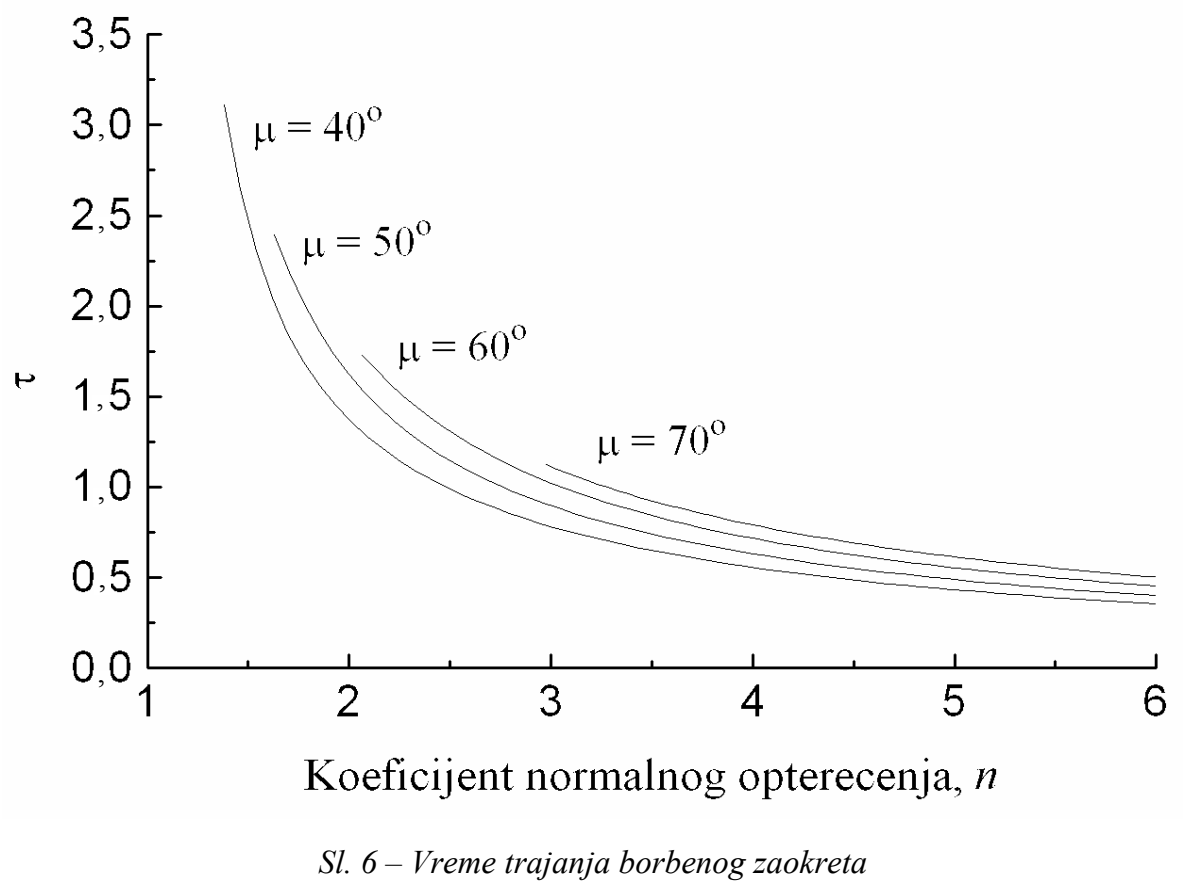




\section{Zaključak}

U radu su izvedene i analizirane jednačine kretanja aviona $\mathrm{u}$ borbenom zaokretu. Jednačine kretanja rešavane su u polubrzinskom koordinatnom sistemu, iz praktičnih razloga, jer se njegovim korišćenjem najlakše dolazi do karakterističnih parametara borbenog zaokreta.

Rezultati proračuna pokazuju:

- vreme zaokreta se smanjuje sa povećanjem koeficijenta normalnog opterećenja i sa smanjenjem ugla naginjanja. Sa povećanjem koeficijenta tangencijalnog opterećenja povećava se vreme zaokreta, ali je taj uticaj umeren i sve manji što je veći koeficijent normalnog opterećenja;

- za prirast visine optimalni uglovi naginjanja su između 45 i $50^{\circ}$ za sve vrednosti koeficijenta normalnog opterećenja. Prirast visine se povećava sa povećanjem koeficijenta $n_{x}$, ali za veće vrednosti koeficijenta normalnog opterećenja uticaj je mali.

\section{Literatura:}

[1] Rendulić, Z.: Mehanika leta, Vojnoizdavački i novinski centar, Beograd, 1987.

[2] Nenadović, M.: Stabilnost i upravljivost letelica - prvi deo, SSNO, Beograd, 1981.

[3] David G. Hull: Fundamentals of Airplane Flight Mechanics, Springer 2007.

[4] Gajić, D.: Mehanika leta - Neustaljena kretanja aviona, Žarkovo, 1986. 